\title{
Pedagogía para la paz en la extensión universitaria: una propuesta lúdica con juegos cooperativos
}

\section{Pedagogy for Peace in the University Extension: \\ a Ludic Proposal with Cooperative Games}

\section{Pedagogia de la Paz na extensão universitária: uma proposta lúdica com jogos cooperativos}

\author{
Evelyn Cerdas-Agüero \\ Coordinadora del Programa Aula Activa \\ Instituto de Estudios Latinoamericanos \\ Universidad Nacional \\ Costa Rica \\ Recibido 30/1/2019 \\ Aceptado. 20/3/2019 \\ DOI:https://doi.org/10.15359/tdna.35-65.15
}

\section{Resumen}

Este texto, por medio de un abordaje cualitativo de análisis teórico y reflexivo, tiene como objetivos promover la educación para la paz como eje pedagógico en la extensión universitaria y generar una reflexión acerca de la trascendencia de incorporar el juego cooperativo en los proceso de extensión en la UNA.

Se concluye que la educación y los procesos grupales se

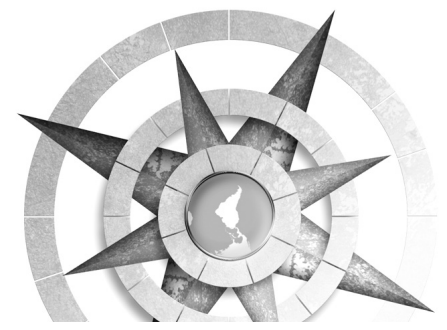


It is concluded that education and group processes can be articulated around the cooperative games as a fundamental axis that allows building collective knowledge, promoting experiences, as well as the development of certain skills to strengthen group processes.

Keywords: cooperative games, education for peace, extension, IDELA, Universidad Nacional

\section{Resumo}

Este texto, por meio de uma abordagem qualitativa de análise teórica e reflexiva, visa promover a educação para a paz como eixo pedagógico na extensão universitária e gerar uma reflexão sobre a importância de incorporar o jogo cooperativo no processo de extensão na UNA. Conclui-se que a educação e os processos de grupo podem ser articulados em torno do jogo cooperativo como um eixo fundamental que permite construir o conhecimento coletivo, promovendo experiências, bem como o desenvolvimento de certas habilidades para fortalecer os processos de grupo.

Palavras chave: jogo cooperativo, educação para a paz, extensão universitária, IDELA, Universidade Nacional

\section{Introducción}

El Instituto de Estudios Latinoamericanos (IDELA), por medio del proyecto de extensión "Aula Activa: Juegos cooperativos para la educación para la paz" ha trabajado en el área de la educación para la paz reconociendo esta como un proceso esencial para la construcción y consolidación de una cultura de paz y de reconocimiento de los derechos humanos. El proyecto se ha implementado como un proceso pedagógico innovador con diversas comunidades y grupos del país, incorporando una metodología participativa en la Educación para la paz que ha privilegiado procesos vivenciales por medio de los juegos cooperativos, propuesta que ha contribuido al trabajo en equipo, liderazgo, solidaridad, integración, toma consensuada de decisiones, diálogo, no violencia, reconocimiento de los derechos humanos y manejo pacífico de los conflictos. Con base en esta experiencia de implementación del proyecto desde el IDELA es que este trabajo, por medio de un abordaje cualitativo de análisis teórico y reflexivo, tiene como objetivos: promover la educación para la paz como una pedagogía en la extensión universitaria; y generar una reflexión acerca de la trascendencia de incorporar el juego cooperativo en los proceso de extensión en la UNA para promover espacios dinámicos, colaborativos y de construcción conjunta.

Se considera importante la incorporación de la de la educación para la paz como eje de la extensión en la Universidad Nacional porque esta promueve 
la participación plena y efectiva de las personas, en las comunidades y en la sociedad en general, genera herramientas para empoderarse y reconocer el rol de cada persona en la construcción de una cultura de paz por medio de la participación en procesos personales, sociales, económicos, políticos y culturales en los diversos espacios en los que se desarrollan. A la vez que permite generar nuevas propuestas conjuntas para construir la paz en la realidad desde la participación y la voz de actores de la sociedad y de la universidad.

La educación para la paz como propuesta pedagógica se dirige al fomento de la cultura de paz por medio de la creatividad, la formación en valores, el, respeto de los Derechos Humanos y las libertades fundamentales, la justicia, la democracia, la comprensión, el diálogo y la solidaridad, por medio de procesos dirigidos a la búsqueda de la transformación de la condición humana y la sociedad, las estructuras sociales y los patrones culturales que promueven la violencia, la injusticia, el irrespeto a los derechos humanos y a la dignidad de los seres humanos.

También es importante el reconocimiento del vínculo de la educación para la paz y los derechos afines que se que se proclaman en la legislación costarricense en el ámbito constitucional, la Constitución Política de Costa Rica (1949) establece en los artículos 1, 12,
20,21, 26, 50 una visión de paz para la ciudadanía relacionada con la democracia, la libertad, la independencia, la no esclavitud, el respeto a la vida, la realización de reuniones pacíficas y la búsqueda del bienestar de las personas. Estos artículos de la Constitución son bastante explícitos, sin embargo, es necesario mencionar que los derechos humanos son interdependientes y el derecho a la paz se visualiza a través de otros Derechos y garantías individuales (Título IV) entre los que se pueden citar la libertad de tránsito (art. 22), el derecho a la propiedad privada y al domicilio privado (art. 23, 24, 45), a la no esclavitud ni tratos crueles (art. 40), a la libertad de expresión e información (art. 28, 29,30) y a la igualdad (art.33).

Así, es necesario reconocer la labor fundamental que tiene la universidad pública, de trabajar en favor de los derechos humanos y la paz como una tarea compleja en la sociedad, por medio del vínculo con esta porque la visión de la Universidad Nacional (UNA) implica el fomento de la comunicación y la colaboración entre los actores de la sociedad, la preparación de las personas y el de las comunidades para su desarrollo, además, contribuye a:

...crear y transmitir conocimiento en favor del bienestar humano, mediante acciones que propician la transformación de la sociedad para llevarla a estadios superiores 
de convivencia. Honra la libertad, la diversidad, la búsqueda de la verdad y la sustentabilidad natural y cultural, en beneficio del conocimiento, la equidad, la justicia y la dignificación de la condición humana. (Estatuto Orgánico, 2015, preámbulo, párr. 1).

Además, la educación para la paz favorece el cumplimiento de los fines del quehacer de la UNA estipulados en el Estatuto Orgánico (2015), como la interdisciplinariedad, en la cual se enfatiza "la articulación permanente entre diversas disciplinas y la búsqueda de su complementariedad" (art. 3. b), el pensamiento crítico para promover "el análisis sistemático y permanente de la realidad nacional e internacional, con el fin de determinar sus tendencias, y a partir de este conocimiento detectar sus problemas, necesidades y fortalezas, para ofrecer alternativas de solución" (art. 3.g) y concuerda con el compromiso de fomentar los principios y valores de la UNA como la inclusión, el conocimiento transformador, el compromiso social, "la justicia, el bien común, el respeto irrestricto a la dignidad humana y a los derechos de las personas y de la naturaleza" (Estatuto Orgánico, 2015, art. 1.a), así como "la orientación de las tareas institucionales hacia el bien común, en particular hacia la promoción y consecución de una mejor calidad de vida para los sectores sociales menos favorecidos" (art. 2 ).
Por otra parte, también es importante la implementación de los juegos cooperativos en los procesos de trabajo grupal, de vinculación y de propuestas colectivas porque estos generan espacios para el reconocimiento mutuo, permiten explorar y facilitar soluciones creativas en un entorno libre, propician relaciones empáticas y de cooperación, la participación, el liderazgo, la comunicación asertiva, el aprendizaje de los errores, la búsqueda consensuada de soluciones y el aprendizaje de valores y habilidades para la convivencia. Los procesos que se generan en el juego privilegian la cohesión grupal, mejores relaciones entre las personas de diversos contextos e instituciones que permite un liderazgo cooperativo, la construcción de espacios de confianza, una visión más amplia de los contextos y la comprensión para el trabajo en equipo. Además, dan paso a que afloren sentimientos, pensamientos desde lo interno de cada persona, como sujeto capaz de mostrar su autonomía, ejercer su libertad de expresión y de reconocimiento de su dignidad como ser humano.

\section{Educación para la paz: una pedagogía para la transformación}

La educación para la paz es un proyecto pedagógico y un derecho humano dirigido a la comprensión entre las personas y los pueblos, el desarrollo cognitivo, actitudinal y afectivo; se enmarca en dos derechos humanos que se entrelazan, la 
educación y la paz. De acuerdo con la Declaración universal de derechos humanos (1948, art. 26) "1.Toda persona tiene derecho a la educación (...)”, según esta misma declaración y el Pacto internacional de derechos económicos, sociales y culturales (1966) la educación debe orientarse a promover el desarrollo del ser humano, el reconocimiento de su dignidad, el respeto por los derechos y libertades fundamentales, la comprensión, la aceptación y la amistad.

En este sentido el derecho a la educación se centra en el ser humano, la interdependencia de sus derechos y sus interacciones, de forma que permita construir relaciones de respeto en las que se valore la dignidad de cada persona y se promueva su calidad de vida, además, suscitan conocimientos y desarrollo de habilidades para la vida. Es así como Freire (1997) manifiesta que la educación es un proceso participativo que busca la transformación de las personas y de la sociedad, la comprensión y la concienciación de la opresión, es, por lo tanto, una educación hacia el empoderamiento y la libertad, para la emancipación. Según Freire (2006), es liberadora al permitir que las personas asuman su compromiso y exista el reconocimiento del sujeto como actor capaz de transformar la sociedad, por ende, es primordial su transformación, la búsqueda de su libertad y el respeto.
La extensión universitaria puede dirigirse a construir, por medio de los componentes de la educación para la paz, mecanismos por medio de los cuales las personas gestionen procesos hacia la transformación individual y colectiva de forma que cada persona se reconozca como gestora del cambio y de la realidad, hacia formas de vida más solidarias y armoniosas que se dirigen a una vida en comunidad más justa.

Cada ser humano puede dirigir sus esfuerzos como parte de una acción solidaria a vivir en paz, en libertad, a manifestarse ante la violencia, la injusticia social, la falta de oportunidades, como lo establece la Declaración universal de derechos humanos (1948) en su artículo 28, en tanto que todas las personas tienen derecho a vivir en paz, de forma que en este se visibilice el respeto a los derechos humanos y las libertades fundamentales. Así la paz se vincula con la forma de vida, con el reconocimiento y la conciencia de la paz como una forma de convivencia que parte del ser humano individual para permear la colectividad por medio de las relaciones sociales entre personas, grupos, pueblos y naciones, es, entonces, la paz un derecho que tiene su realización en la praxis y este debe verse reflejado en la interdisciplinariedad con la cual se trabaja en la universidad.

La paz, se constituye como condición fundamental para la realización de los 
derechos humanos, su respeto y reconocimiento, implica una labor colectiva, contextualizada, intergeneracional para su realización y consolidación, la cual no puede estar invisibilizada por el trabajo de la universidad con la sociedad, en tanto, esta como institución busca el bien común e incidir en la realidad para que esta sea más justa, libre y democrática. Desde esta perspectiva se considera la paz como un proceso inacabado, en construcción, una "paz imperfecta" como proceso permanente, de búsqueda constante "que intenta construirse día a día en todos los escenarios, facilitando el análisis de ideas, valores, actitudes y conductas relacionadas con la paz" (Martín, 2015, p. 243); esta vivencia constante es en la cual la UNA puede incidir en espacios donde se aprecien las acciones que crean paz y que combaten todas aquellas acciones e inacciones que la limitan.

La propuesta de la paz imperfecta implica que no hay un estado de paz, absoluta y perfecta, sino, un proceso que se va descubriendo y construyendo cada día en las prácticas que llevan a cabo las personas, las instituciones y en general los diferentes actores de la sociedad, pero para esto es necesario promover este espacio de reconocimiento, sensibilización, empoderamiento ante esta imperante necesidad, que a la vez es un reto ante la pasividad y la naturalización de las diversas formas de violencia. En este sentido se habla de un proce- so que permite tomar conciencia de las personas, la sociedad y los diversos contextos para construir la realidad de forma conjunta, cooperativa y promover una convivencia pacífica como "(...) un proceso por el cual los seres humanos se van autoconstruyendo en comunicación con otros seres humanos (...)" (López, 2006, p. 9 citado por Gómez, 2010, p. 126), incluye el desarrollo de habilidades, la reflexión, la búsqueda de soluciones de problemas, la cooperación, la resolución de conflictos y la búsqueda de oportunidades para la transformación de la condición humana (Harris y Lee, 2013).

Así, se propone una pedagogía para la vida, la libertad, el respeto por los derechos humanos y el reconocimiento de la dignidad intrínseca de cada ser humano hacia la construcción de relaciones más armoniosas y comprometidas con la incidencia en nuevas formas de vivir en sociedad, que a la vez implica construir de forma conjunta un proceso dinámico que no es neutral tanto desde la universidad como desde las comunidades, lo cual quiere decir que la extensión debe fundamentarse en determinados valores y principios que buscan incidir en el cambio social, estimular la reflexión crítica ante la realidad y asumir opciones ante las condiciones injustas, opresoras y de violencia.

Es importante reconocer que la universidad en su trabajo de acción e incidencia 
en la sociedad por medio de la extensión puede priorizar aprendizajes planteados como pilares de la educación para construir la paz, los cuales, además, se constituyen en bases importantes para construir una sociedad más justa, con más oportunidades en la que se busque el bien común, lo cual implica un compromiso social e individual para la trasformación de la sociedad, estos pilares fueron señalados por Delors (1996) aprender a ser, aprender a vivir juntos, aprender a hacer y aprender a conocer. Estos aprendizajes, pilares del desarrollo de una pedagogía para la paz implican un cambio profundo en las personas, los grupos y la sociedad en general, hacia una pedagogía socialmente crítica, holística y política según plantea García (2016), un proceso que se puede construir desde la universidad con las comunidades, implica la interiorización de valores, la adquisición de conocimientos y el desarrollo de destrezas en entornos participativos en los que se genere la reflexión; el abordaje de los conocimientos y aprendizajes de forma integrada con sus vivencias y las situaciones de la realidad social como aquellas vinculadas a los derechos humanos. También, se vincula con la "deslegitimación de la violencia en la sociedad como herramienta política" (p.47) y con el compromiso individual y colectivo para reconocer las formas de violencia e incidir en estas para transformar la condición humana e incidir en el respeto a la dignidad de cada ser humano.

\section{El juego cooperativo: espacio de en- cuentros y vivencias}

El juego es una actividad que ha estado presente en el desarrollo del ser humano y ha privilegiado sus relaciones desde su nacimiento, lo cual lo ha hecho indispensable en su devenir. A la vez se constituye imprescindible, tanto para la persona como para la comunidad, porque tiene sentido, significado, valor expresivo e implica conexiones espirituales y sociales, funciones culturales de expresión y de convivencia.

Estas características hacen que este sobrepase lo habitual, el juego de acuerdo con Huizinga (2000) no se refiere a vida ordinaria sino que es la recreación de otra, que puede ser imaginada, construida en el proceso y que también puede reflejar elementos de la realidad, "El juego no es la vida «corriente» o la vida "propiamente dicha». Más bien consiste en escaparse de ella a una esfera temporera de actividad que posee su tendencia propia” (p.21), así, tiene una función primordial en el desarrollo humano ya que recrea y refleja parte la realidad a la vez que implica libertad, sentido, sucede en sí mismo y está fuera de la vida corriente, pero no implica que no pueda gestionar su transformación, conforman un modelo de sociedad en la cual se dan diversas relaciones que tienen sentido al ser trasladadas a la realidad y a las interacciones humanas en diversos contextos, a esto Miranda 
(2008) lo cataloga como una minisociedad en la cual se genera la formación de valores hacia la solidaridad y la cooperación, lo que implica que el juego en el acercamiento grupal y comunitario dinamiza los proceso de interacción social que amplía la convivencia.

De tal forma que los juegos cooperativos se refieren a juegos (actividades lúdicas) no tradicionales que buscan generar espacios vivenciales de aprendizaje caracterizados por la participación, la diversión y la cooperación basados en la libertad y el respeto a la dignidad humana, se refieren a:

(...) propuestas que buscan disminuir las manifestaciones de agresividad en los juegos promoviendo actitudes de sensibilización, cooperación, comunicación y solidaridad. Facilitan el encuentro con los otros y el acercamiento a la naturaleza. Buscan la participación de todos, predominando los objetivos colectivos sobre las metas individuales. Las personas juegan con otros y no contra los otros; juegan para superar desafíos $\mathrm{u}$ obstáculos y no para superar a los otros (Pérez, 1998, p.1).

En este proceso las personas participantes forjan metas comunes, lo cual implica un sentido de cooperación, eliminado el espacio para la competencia, el individualismo o la exclusión sino la participación, es un espacio de encuentro que se puede privilegiar en los procesos de extensión.

De acuerdo con Omeñaca y Ruiz (2005) el juego es fuente de alegría, de júbilo, de placer, constituye un fin en sí mismo, es espontáneo, voluntario y libre, promueve aprendizajes, es una forma de expresión, implica participación activa, es un espacio de encuentro con la realidad, es un mundo aparte en el cual se van construyendo y recreando experiencias, aprendizajes y visiones de mundo en el que las personas son el centro del proceso lúdico del juego con sus conocimientos, habilidades, capacidades, historia y necesidades y produce interiorización, reflexión, análisis, introspección, este implica, además, un proceso personal, de reflexión, interiorización, actitudinal, cognoscitivo.

Además, privilegia las vivencias, generan un proceso de aprendizaje en el cual se promueve que las personas compartan, se relacionen, aprendan de los errores, se conozcan, resuelvan y manejen conflictos, se genere un clima de confianza, practiquen técnicas de comunicación asertiva, se enfoquen en ganar-ganar, fortalezcan la toma de decisiones, se generen espacios de organización y planteamiento de estrategias. Garaigordobil (2003) plantea que el juego contribuye con diversos aspectos tales como el intelecto, porque jugando se aprende y se tienen nuevas experiencias, se ponen 
en práctica conocimientos, propuestas para solucionar problemas, se cometen errores y aciertos, además, estimula el pensamiento, la generación de ideas, propuestas y la creatividad; la sociabilidad, permite un espacio para encontrarse con iguales, conocer a las personas que le rodean, reconocer, aceptar y respetar las diferencias y semejanzas; el desarrollo afectivo y emocional, el juego genera placer, alegría, diversión, libertad y espacios de expresión libre y para descargar tensiones.

Asimismo, forman parte de la metodología participativa, la cual es una forma de abordar los procesos pedagógicos, de construcción, planeación y evaluación conjunta, elementos esenciales en la extensión; además, se caracteriza por ser lúdica, interactiva, creativa y flexible, además, fortalece la cohesión grupal, también, es formativa, porque da prioridad a los procesos, promueve el compromiso entre las personas participantes y se caracteriza por ser vivencial.

\section{Educación para la paz en la extensión universitaria}

Como propuestas se visibiliza la posibilidad de incorporar en la extensión universitaria dos ejes: la educación para la paz como proceso de transformación que permite construir, promover y consolidar una cultura de paz; y el juego cooperativo como espacio y proceso de diálogo de experiencias y vivencias, desarrollo de habilidades y de empatía para la construcción grupal.

La educación para la paz se enfoca en la promoción de relaciones solidarias, la convivencia pacífica, las alternativas para la resolución pacífica de conflictos el compromiso en la transformación como parte de la praxis cotidiana y permite generar una conciencia del rol que cada persona tiene en su comunidad como eje de incidencia y transformación, este rol es importante porque la educación para la paz es también una propuesta política, porque busca la transformación hacia la justicia social. En este sentido se debe plantear la extensión como un proceso que permita empoderar a las personas desde sus realidades y contextos para que sus relaciones, proyecto de vida, iniciativas y cotidianeidad tenga como marco de referencia la construcción continua de la paz y el reconocimiento de los derechos humanos y de la dignidad humana.

Los juegos cooperativos como técnicas lúdicas en la metodología participativa son importantes en los proceso de extensión de la universidad porque promueven procesos centrados en una visión de comunidad en la cual se construyen aprendizajes y propuestas de transformación de la sociedad, dentro de una praxis solidaria en la que se reconoce la libertad y los derechos, así como la cooperación entre las personas, grupos, comunidades y organizaciones 
en un ámbito de reconocimiento de la co-responsabilidad hacia una mejor calidad de vida y respeto de la dignidad del ser humano. Esto es, precisamente, lo que busca la educación para la paz también y a lo que el juego cooperativo contribuye, facilitar procesos sociales centrados en la solidaridad, la confianza, el respeto mutuo y la comprensión.

Así la educación para la paz puede incidir en la extensión universitaria:

- Como proceso de capacitación permanente que permita reconocer y vivenciar las diversas técnicas que trascienden lo tradicional y dan lugar a la participación, la afectividad, el encuentro a través del juego y de las actividades participativas.

- Como una visión de procesos participativos, hacia la construcción colectiva, la escucha mutua y el diálogo.

- Al promover las interacciones y las relaciones humanas afectivas, respetuosas, de confianza en las comunidades como parte de la convivencia pacífica.

- Dirigirse a la construcción de espacios y proyectos que aborden la prevención de la violencia.

- Integrar propuestas hacia un proyecto local y nacional contextualizado de educación para la paz formal y no formal.

- Promover la afirmación, el empoderamiento, la autonomía de cada persona, en tanto sujeto social de dere- chos que cumple un rol importante en la sociedad y consigo mismo.

- Promover el derecho a la paz.

Por otra parte, las características que posee el juego cooperativo le dan un valor único en el contexto de trabajo con grupos y comunidades puesto que genera situaciones de interacción social en las que se manifiestan actitudes y toma de decisiones, que reflejan las realidades de cada persona, grupo y contexto.

Además, representa dinamismo, es un entorno en el cual se dan interacciones, relaciones, y diversos eventos que son parte de un proceso que se va construyendo, es decir el juego permite construir procesos de toma de decisiones, solución de conflictos, diálogo, confianza, consenso, propuestas y de puesta en común de metas, objetivos y realidades, los cuales son necesarios en la extensión universitaria.

Asimismo, permite construir aprendizajes en el proceso lúdico por medio de la interacción individual, en lo que cada uno conoce y experimenta, la propia historia; grupal, las interacciones que se dan entre los miembros del grupo o comunidad; y las interacciones con el entorno ya sea social, político, económico, histórico u natural. 
Los juegos cooperativos como parte de la lúdica pueden constituirse como una vivencia, un espacio gestor y un proceso que permite dentro de la extensión:

- Promover el diálogo entre extensionistas que permita conocer sus capacidades, realidades, necesidades y propuestas.

- Desarrollar y fortalecer las capacidades de gestión y las habilidades en ámbitos de competencia para el trabajo con las comunidades y grupos tales como la comunicación asertiva, el diálogo, el liderazgo cooperativo, la cooperación, el manejo de los conflictos.

- Desarrollar un espíritu crítico y reflexivo con respecto a la potencia del juego en el trabajo comunitario y sus posibilidades.

- Desarrollar las habilidades y competencias en los grupos comunitarios para reflexionar acerca de sus conclusiones sobre las acciones y vivencias experimentadas con los proyectos de extensión y a través de estos.

- Propiciar los espacios de diálogo y reflexión en las comunidades, de forma que sean placenteros, afectivos, alegres, cuya base sea el respeto, la participación y el compromiso.

- Promover las interacciones y las relaciones humanas afectivas, respetuosas, de confianza en las comunidades como parte de un proceso lúdico.
- Desarrollar por medio del juego la expresión y la comunicación en sus diversas dimensiones, en tanto derecho humano y como elemento necesario en la gestión y trabajo conjunto para el desarrollo de acciones.

- Abordar la vivencia plena e integral de diversos temas que se pueden abordar.

- Privilegiar el trabajo en equipo.

\section{¿Qué se necesitaría hacer para su im- plementación?}

Procesos de capacitación y creación de espacios con extensionistas que permitan:

- El conocimiento, la reflexión crítica y vivencia de la educación para la paz y los temas afines a esta involucrando el componente cognitivo, actitudinal y afectivo.

- El reconocimiento de diversas posibilidades de educación para la paz desde la interdisciplinariedad de forma que se logre poner en común diversos lenguajes y saberes que permitan reconocer la trascendencia de pedagogías para la paz interdisciplinarias.

- Procesos de reflexión y planteamiento de propuestas basadas en el diálogo, la cooperación hacia la construcción de una cultura de paz desde el reconocimiento del derecho humano a la paz que permitan incidir en algunas a necesidades sociales. 
El reconocimiento, de forma vivencial y con aportes teóricos, de la potencia del juego cooperativo y las posibilidades de este en el trabajo con grupos y en el abordaje comunitario.

La importancia del juego como una forma metodológica participativa en los procesos de aprendizaje y como actividad sociocultural.

Promover la construcción de proyectos interdiciplinarios que permitan desarrollar propuestas pedagógicas para la paz.

\section{Conclusiones}

Este trabajo es resultado de la práctica y de las experiencias con diversos grupos a través del proyecto de extensión "Aula Activa: juegos cooperativos para la educación para la paz" del IDELA y de un abordaje teórico por medio del cual se justifica, reflexiona y plantea la importancia del juego en la extensión, pero además, de una puesta en práctica de la educación para la paz como eje pedagógico, elementos que se conjugan para la construcción de una cultura de paz.

Cabe recalcar la importancia, de que tanto en la educación para la paz como en el juego cooperativo, la universidad por medio de la extensión está dando el protagonismo a la comunidad, tanto, concebida esta como espacio social, y también como grupo, ante lo cual se acerca a su realidad, historia, conocimientos, sentimientos, vivencias y actitudes, que no pueden escapar de su contexto. Esta realidad se presenta ante los ojos del extensionista en el momento y en el proceso de encuentro con esta, que no es más que un espacio dinámico, vivencial en el cual se deben proporcionar los espacios participativos que permitan la incidencia mutua, el conocimiento y el trabajo conjunto de forma horizontal.

El juego cooperativo reconoce la creación conjunta de un espacio para el análisis y la reflexión de diversas posibilidades de desarrollo de la persona y del grupo como las afectivas, cognitivas, actitudinales, sociales, de aprendizaje, a la vez que promueve y genera procesos participativos e integradores en los que todas las personas tiene voz y plantean propuestas, es decir, una actitud hacia la búsqueda de acciones, propuestas colectivas en las que se incorpora las experiencias personales. Además, transmite, recrea y fortalece tradiciones, creencias, costumbres, conductas sociales y actitudes que son parte del desarrollo cultural de una comunidad o grupo.

Por otra parte la educación para la paz promueve interacciones grupales en las que se privilegian el conocimiento mutuo, las relacione positivas y no violentas, la cooperación, la comunicación asertiva y no-violenta, el sentido de pertenecía, la cohesión del grupo y el manejo pacífico de los conflictos, aspectos fundamentales en la vivencia y 
construcción de una cultura de paz que deben acompañar la labor de la extensión universitaria con las comunidades.

\section{Referencias bibliográficas}

Agrelo, A. (sf.). Metodología participativa. Recuperado de http:// es.slideshare.net/andreagrelo/ metodologa-participativa

Asamblea General de las Naciones Unidas. (1966). Pacto Internacional de Derechos Económicos, Sociales y Culturales. Consultado en: http://www2.ohchr.org/spanish/law/cescr.htm

Asamblea General de Naciones Unidas (1948). Declaración universal de derechos humanos, A/RES/217A/ III. Recuperado de: http://www. ohchr.org/EN/UDHR/Pages/ Language.aspx? LangID $=$ spn

Asamblea General de Naciones Unidas (1978). Declaración sobre la preparación de las sociedades para vivir en paz, A/RES/33/73. Recuperado de: http://daccess-dds-ny. un.org/doc/RESOLUTION/ GEN/NRo/367/12/IMG/ NR036712.pdf?OpenElement

Asamblea Legislativa de la República de Costa Rica (1949). Constitución política de Costa Rica. Recuperada de http://www.constitution. org/cons/costaric.htm.
Asamblea Legislativa de la República de Costa Rica. (1997). Ley sobre resolución alterna de conflictos y promoción de la paz social. Ley N. 7727. Recuperado de http:// www.derpublico.net/main.php/ view_photo?wa_id=13

Delors, J. (1996). La educación encierra un tesoro. Madrid: Santillana. Ediciones UNESCO.

Freire, P. (1997). Pedagogía de la autonomía. Buenos Aires: Siglo XXI editores.

Freire, P. (2006). Pedagogía de la esperanza. Buenos Aires: Siglo XXI editores.

Garaigordobil, M. (2003). Diseño y evaluación de un programa de intervención socioemocional para promover la conducta prosocial y prevenir la violencia. España: CIDE.

Garaigordobil, M. (2005). Diseño y evaluación de un programa de intervención socioemocional para promover la conducta prosocial y prevenir la violencia. España: CIDE.

García, E. (2016). Educar para la paz desde los derechos humanos y la justicia social. En: Villar, C.; García, E; Martín, V.; Ramírez, C. (Coord.). Estudios en cultura de paz, conflictos, educación, y derechos humanos. Madrid: Editorial Síntesis. Pp.45-74. 
Gómez, M. E. (2010). La Educación para la paz aplicada a la tutoría académica en la Facultad de Ciencias Políticas y Sociales de la Universidad Autónoma del Estado de México. Revista Paz y Conflictos, 3, 2010, 123-139. Recuperado de http://www.ugr.es/ revpaz/ tesinas/rpc_n3_2010_dea3.pdf

Harris, I. \& Lee, M. (2013). Peace education. North Carolina: McFarland \& company, Inc., Publishers.

Huizinga, A. (2000). Homo ludens. Barcelona: Alianza editorial.

Martín, V. (2015). Derechos humanos, ciudadanía crítica y cultura de paz. encuentros ontológicos desde la educación social. En Coca, C.; García, E.; Martín, V. \& Ramírez, C. (coords.), pp. 241-256. Estudios en cultura de paz, conflictos, educación y derechos humanos. España: Editorial Síntesis.

Miranda, M. (2008). Trabalhando com jogos cooperativos: em busca de novos paradigmas na educação física. Sao Paulo: Papirus Editora. 2 edic.

Omeñaca, R. y Ruiz, J. (2005) Juegos cooperativos y educación física. Barcelona: Editorial Paidotribo.
Pérez, E. (1998).Juegos cooperativos: juegos para el encuentro. Educación Física y deportes. Año 3, $\mathrm{N}$ o 9.Buenos Aires. Recuperado de http://www.efdeportes.com/ efd9/jue9.htm

UNESCO. (1974). Recomendación sobre la educación para la comprensión, cooperación y la paz internacionales y la educación relativa a los derechos humanos y las libertades fundamentales. Recuperada de http://portal.unesco.org/es/ ev.php-URL_ID $=13088 \&$ URL DO=DO_TOPIC\&URL_SECTION=201.html

Universidad Nacional (2015). Estatuto Orgánico, Universidad Nacional. Recuperado de: http://documentos.una.ac.cr/bitstream/ handle/unadocs/6693/ES. TATUTO-ORG\%C3\%81NICO-UNA-digital.pdf?sequence $=1 \&$ is Allowed $=y$ 